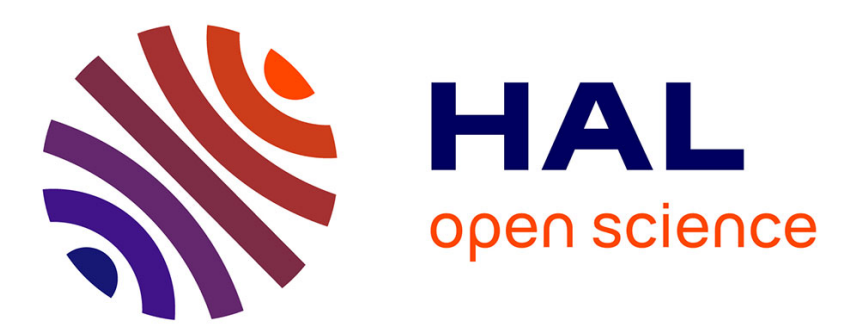

\title{
Contribution de Jean-Marie Privat et Marie-Christine Vinson
}

\author{
Jean-Marie Privat, Marie-Christine Vinson
}

\section{To cite this version:}

Jean-Marie Privat, Marie-Christine Vinson. Contribution de Jean-Marie Privat et MarieChristine Vinson. Pratiques: linguistique, littérature, didactique, 2000, 105, pp.225-232. 10.3406/prati.2000.2414 . hal-03180252

\section{HAL Id: hal-03180252 \\ https://hal.univ-lorraine.fr/hal-03180252}

Submitted on 24 Mar 2021

HAL is a multi-disciplinary open access archive for the deposit and dissemination of scientific research documents, whether they are published or not. The documents may come from teaching and research institutions in France or abroad, or from public or private research centers.
L'archive ouverte pluridisciplinaire $\mathbf{H A L}$, est destinée au dépôt et à la diffusion de documents scientifiques de niveau recherche, publiés ou non, émanant des établissements d'enseignement et de recherche français ou étrangers, des laboratoires publics ou privés. 


\section{Contribution de Jean-Marie Privat et Marie-Christine Vinson} Jean-Marie Privat, Marie-Christine Vinson

\section{Citer ce document / Cite this document :}

Privat Jean-Marie, Vinson Marie-Christine. Contribution de Jean-Marie Privat et Marie-Christine Vinson. In: Pratiques : linguistique, littérature, didactique, n¹05-106, 2000. pp. 225-232;

doi : https://doi.org/10.3406/prati.2000.2414

https://www.persee.fr/doc/prati_0338-2389_2000_num_105_1_2414

Fichier pdf généré le 13/07/2018 


\section{Contribution de Jean-Marie Privat et Marie-Christine Vinson,} Université de Metz et IUFM de Lorraine

\section{Pratique culturelle, pratique cultivée et pratique scolaire}

Si écrire est une pratique culturelle, réécrire est une pratique... cultivée. D'où la nécessité d'introduire d'emblée une double distinction, au double sens du terme.

S'il convient d'entendre ici par « écrire » une praxis scripturale construite dans un processus long d'acculturation à la raison graphique légitime, on se doit de constater qu'elle n'échappe pas aux stratifications sociales contemporaines en matière de pratiques culturelles : « minoritaires, élitaires et cumulatives» (1). Ainsi « plus de sept Français sur dix par exemple n'ont jamais assisté de leur vie à un concert de musique classique, plus de la moitié ne sont jamais allés au théâtre [...] » alors que « plus de la moitié des cadres ont une fréquentation régulière des équipements culturels (salles de cinéma, salles de spectacles, bibliothèques, lieux d'exposition, lieux de patrimoine), soit près de deux fois plus que les professions intermédiaires et cinq fois plus que les ouvriers » (2).

Le sociologue classe l'écriture dans les «pratiques artistiques en amateur » et distingue deux items (qui sont en consonance avec les pratiques d'écriture scolaires réelles ou encouragées) : « tenir un journal, noter des réflexions », "écrire des poèmes, nouvelles ou romans ॥.

Malgré les progrès de la scolarisation, le développement du temps libre, la nécessité de plus en plus ressentie de trouver des activités favorisant la construction de soi et l'expression identitaire, l'évolution et l'intensité des pratiques libres (non scolaires) d'écriture demeurent très faibles. Ainsi seulement $6 \%$ des person-

(1) L'analyse s'appuie sur la dernière enquête nationale statistique sur le sujet. Voir O. Donnat, Les Pratiques culturelles des Français. Enquête 1997, Paris, La Documentation Française, 1998. On rappellera simplement que d'un point de vue méthodologique l'étude repose sur un sondage auprès d'un échantillon représentatif de la population française de 15 ans et plus.

(2) Pour un commentaire sociologique rapide des principaux résultats, se reporter à O. Donnat, « La stratification sociale des pratiques culturelles et son évolution 1973-1997 », Revue française de sociologie, janvier-mars 1999, XI-1, pp. 111-119. 
nes interrogées continuent à déclarer écrire de la fiction par intérêt personnel. Ajoutons que le libre investissement scriptural est d'autant plus inégal et faible que l'on est un homme âgé, peu doté en capitaux culturels légitimes, et habitant à la campagne.

La relative maîtrise pratique de l'écriture acquise assez généralement à l'école n'est donc pas un facteur suffisant pour se transformer en pratique culturelle significative généralisée dans l'espace privé.

Toutefois l'inégalité de pratique est d'autant plus important qu'on ne saurait réduire «l'écriture » à ses usages fictionnels et enjeux lettrés, dans leur version scolaire ou mondaine. En effet, on ne doit pas oublier que dans certaines catégories sociales et dans certaines situations le recours à l'écrit fonctionnel domestique et/ou professionnel est disqualifié : "Lié à sa machine et à ses collègues de travail, pris dans un mode mimétique d'incorporation du travail, l'ouvrier n'est amené à lire et à écrire qu'en de rares occasions. L'écriture n'est perçue que dans sa fonction mnémotechnique [...] comme une sorte de palliatif à une mémoire déficiente. Les pratiques d'écriture peuvent donc être perçues négativement par ceux qui répondent fièrement qu'ils n'ont pas besoin de cela pour le moment, comme s'il avait été question d'une paire de lunettes venant compenser une baisse de vue ou d'une canne pour s'aider à marcher. Utiliser l'écrit marquerait ainsi l'existence d'un handicap, d'une difficulté » (3).

Ces réticences aux usages "fainéants » de l'écriture sont inégalement réparties entre les sexes : "Les femmes "dépassent" les hommes sur l'ensemble des actes d'écriture domestique $"$.

Les femmes des milieux populaires peuvent même être de fait « de véritables machines à écrire familiales ». Cependant, certaines mères de famille "à temps plein " qui ont constamment en tête tous les soucis du groupe familial peuvent ne pas éprouver (sinon désapprouver) le besoin d'écrire. "J'ai une bonne mémoire », dira l'une ; " J'ai pas besoin de noter, j'ai ma tête », dira l'autre (4).

Dans un tel contexte socio-culturel, on peut faire l'hypothèse que si l'acte même d'écrire est loin d'être toujours valorisé, la réécriture a toute chance d'être considérée par certains comme un " sur-travail » littéralement sans objet, quitte à concéder que des écrivains dont c'est le métier ou des gens dont c'est la passion peuvent trouver du plaisir et de l'intérêt à biffer, raturer, ajouter, brouillonner, etc.

Cette conception de l'écriture à l'économie (qui peut aller jusqu'à faire l'économie de l'écriture) est souvent redoublée par la représentation de l'écriture lettrée comme don accordé mystérieusement à quelques privilégiés. Dans cette perspective romantique et aristocratique de la création littéraire comme acte original et inspiré, réécrire est impensable et indu : l'écrivain exprime sa subjectivité sous la dictée de son génie et réécrire serait introduire de l'artificiel, du cérébral, de l'inauthentique (5).

Cette mythologie se nourrit de la censure que les écrivains eux-mêmes peuvent trouver intérêt à exercer sur la genèse de leur œuvre : "Mène-t-on la foule dans les ateliers de l'habilleuse et du décorateur, dans la loge de la comédienne? Montre-t-on au public le mécanisme des trucs ? Lui explique-t-on les retouches et les variantes improvisées aux répétitions [...] ? Lui révèle-t-on toutes les loques,

(3) B. Lahire, "Les pratiques ordinaires d'écriture en action », L'Homme pluriel, Paris, Nathan, 1998, pp.139-142.

(4) B. Lahire, La Raison des plus faibles. Rapport au travail, écritures domestiques et lectures en milieux populaires, Lille, P.U.L., 1993

(5) Il est possible que dans la culture occidentale le mythe chrétien des Saintes Ecritures comme discours inspirés d'origine divine constitue le paradigme sacré du modèle scriptural où le brouillon est (théologiquement) impensable. 
les fards, les poulies, les chaînes, les repentirs, les épreuves barbouillées, etc. " (6).

Autrement dit, il y a tout lieu de penser que réécrire se heurte à la fois à la mise en rapport avec des usages sociaux ordinaires mais aussi avec des représentations idéologiques (semi-)savantes. De plus, il faudrait s'assurer que la réticence de nombre d'élèves à la réécriture ne réside pas aussi dans la concurrence que la culture iconique fait peser sur la culture graphique comme culture désespérément lente, fatalement séquentielle, nécessairement malthusienne. Songeons par exemple à la description romanesque à qui les jeunes générations refusent de plus en plus explicitement toute légitimité esthétique ou pragmatique. Et il conviendrait de leur apprendre non seulement à décrire mais aussi à "redécrire », labeur s'il en fût?

Cette impatience et cette insatisfaction ne sont peut-être que la forme actuelle de la proclamation « artiste » de la supériorité de l'image sur l'écrit : " J'ai sous les yeux des oiseaux qui se baignent dans une petite flaque d'eau qui se forme quand il a plu sur le plomb qui recouvre la saillie plate d'un toit, je vois à la fois une foule de choses que non seulement le poète ne peut pas même mentionner, loin de les décrire, sous peine d'être fatigant et d'entasser des volumes pour ne rendre encore qu'imparfaitement » (7).

En tant qu'élèves, les jeunes scripteurs sont d'autant moins prédisposés à accepter de considérer l'écriture comme un travail de production discursive qui doit passer par des étapes de retravail et de finition avant d'obtenir un produit final plus satisfaisant que leur imaginaire scriptural a rarement l'occasion d'entrer en conflit avec la réalité des processus de réécriture. Ils sont en effet confrontés beaucoup plus souvent à la lecture de produits scripturaux finis qu'à des work in progress et la réécriture (pas plus que la relecture d'ailleurs) n'est que rarement l'objet d'un apprentissage didactiquement organisé (ni d'une pratique régulière parmi le corps enseignant...).

D'autre part, la conception idéaliste de l'écriture, omniprésente jusque dans la doxa scolaire - on cherche ses idées puis on met en forme et si possible des formes - rend relativement gratuit, formel très exactement, le retour sur son texte.

II n'est pas exclu enfin, qu'outre l'économie que les élèves peuvent penser trouver plus ou moins logiquement à ne pas s'engager dans un retravail scriptural aux gains improbables et aux modèles incertains, ils résistent à la réécriture pour des raisons positives assez compréhensibles : reprendre son texte c'est accepter l'idée que la réécriture peut être sans fin (perspective anxiogène s'il en est sur le marché scolaire !) et surtout accepter de dévoiler son intimité textuelle à un tiers. Les reprises, les retouches, les retours, bref, les modifications apportées à un état antérieur de son propre texte, mettent comme à nu les hésitations, les choix, les partis pris, jouant en somme le rôle d'un verre grossissant de la personnalité profonde et privée du scripteur.

Comment aider les jeunes scripteurs à se défaire de leur langue de bois, gangue où le moi scolaire se protège et se nécrose, s'il est vrai que « la vie sociale est dans l'écriture » (8)?

De nos observations historiques, sociologiques et ethnologiques, il découle plusieurs pistes didactiques.

(6) Ch. Baudelaire, L'Art romantique.

(7) E. Delacroix, Journal, 16 décembre 1843.

(8) D. Fabre, Par écrit. Ethnologie des écritures quotidiennes, sous la dir., Paris, Ed. de la Maison des sciences de I'homme, Mission du Patrimoine ethnologique, Collection Ethnologie de la France, Cahier 11, 1997, pp. 2-3. 


\section{La réécriture observée}

Dans une phase de la séquence de réécriture, il serait pertinent de mettre les élèves dans des situations qui les aident à accepter d'entrer dans un rapport plus expert à la production textuelle.

Donnons ici deux exemples de situations scolaires où les élèves peuvent observer le travail « professionnel » de réécriture.

La critique génétique contemporaine s'exerce sur des avant-textes qui constituent en somme le dossier d'écriture d'une œuvre littéraire : esquisses, ébauches, scénarios partiels, plans d'ensemble, plans topographiques, résumés partiels, notes marginales, amorces de phrases ou de paragraphes, brouillons, biffures, ratures, signes d'appel, mises au net, variantes. Si l'on admet en effet que le texte, dans toute sa perfection formelle, "reste le résultat d'un travail " et que l'œuvre est avant tout « l'effet de sa propre genèse », l'étude des manuscrits permet d'observer « le texte en travail » (9).

Les élèves n'ont pas vocation à devenir des généticiens de la textualisation; on les introduira dans l'atelier de l'écrivain en se contentant de les sensibiliser au bricolage textuel (bris et collage parfois aussi) par le repérage du jeu des quatre figures cardinales de l'opération de réécriture : suppression, permutation, substitution, adjonction (10). Ils pourront constater de visu qu'en deçà de sa clôture formelle le texte d'une œuvre publiée est, à de très rares exceptions près, " le résultat d'une construction progressive, de diverses campagnes d'écriture " (11). On se rendra compte aussi que la réécriture peut être relancée par un « dossier de rédaction " qui convoque notes de lecture complémentaires, correspondance ou journal littéraire de l'auteur, recherches d'informations sur le terrain, documents iconographiques, etc. Ainsi le regard se déplacera-t-il des « questions du sens ou de la forme » pour se porter « sur les problèmes du processus, de l'invention, de la technique » (12).

II est clair que ces modèles ont leurs limites dans la mesure où, précisément, ces modèles sont ceux de pratiques d'experts que les élèves peuvent fort bien considérer comme inaccessibles à des apprentis et liées à des situations, des temps et des enjeux d'écriture extra-ordinaires. Mais, inversement, ils peuvent tout aussi bien prendre acte que même les auteurs les plus légitimes pratiquent la réécriture.

Les ethno-textes fournissent un autre excellent support de réflexion sur les procédés de réécriture, dans un autre contexte d'énonciation (13). II s'agit de faire un livre à partir d'un entretien oral avec un témoin, souvent d'origine ou de condition populaire, qui raconte sa vie : le texte produit présente des marques codées d'oralité (interjections, temps, personnes, vocabulaire, etc.) qui coexistent avec des marques caractéristiques de l'écrit académique (double négation, subordination, absence de répétition, effacement des hésitations, etc.).

Dans ce genre de discours, le scripteur n'est pas le locuteur même s'il assure la

(9) P.-M. de Biasi, « L'avant-texte », Le Grand Atlas Universalis des Littératures, Encyclopaedia Universalis France, 1990, pp. 24-25.

(10) On pourra se reporter à notre contribution, J.-M. Privat et M.-C. Vinson, "Scriptor in fabula », Les Interactions lecture-écriture, éd. Y. Reuter, Peter Lang, 1994, pp. 243-261.

(11) P.-M. de Biasi, op. cit.

(12) Id, ibid. De Montaigne à Proust, en passant par Hugo et Flaubert, les éditions disponibles des avant-textes littéraires français sont nombreuses depuis quelques années, parfois sous forme de cédéroms avec possibilité de navigation hypertextuelle (voir par exemple la collection Manuscrits, CNRS Editions).

(13) Les récits de vie ethno-textuels sont souvent édités en livres de poche. A titre d'exemple

E. Grenadou et A. Prévost, Grenadou, paysan français, Paris, Le Seuil, 1966. E. Carles, Une soupe aux herbes sauvages, Paris, Ed. J.-Cl. Simoën, 1977. V. Jameray-Duval, Mémoires, Enfances et éducation d'un paysan au $X V I I I^{e}$ siècle, présentés par J.-M. Goulemot, Paris, Le Sycomore, 1981. C. Rivals, Pierre Roullet, la vie d'un meunier, Marseille, J. Laffitte, 1983. 
réécriture de la transcription brute des entretiens enregistrés. Le travail de réécriture (de conversion discursive en l'occurrence) s'observe assez aisément si l'on compare dans un premier temps la transcription d'un entretien oral avec une page de récits de vie et si dans un deuxième temps l'on compare une page de l'un de ces ethno-textes avec un récit littéraire.

Ce double travail de comparaison met en évidence une rhétorique qui procède de l'oral vers un écrit... qui vise à connoter l'oral.

Il va de soi que ces prises de conscience de la dynamique écriture-réécriture n'ont de sens que si le travail présente un intérêt que l'élève peut s'approprier.

\section{La réécriture différée}

On peut faire pratiquer cette fois directement la réécriture, sur un mode relativement jubilatoire ou gratifiant, en faisant jouer les compétences nouvelles normalement acquises au fil des ans. Ainsi, des élèves de $3^{\mathrm{e}}$ par exemple (ou de terminales) réécrivent après coup des textes qu'ils ont eux-mêmes produits ou que $\mathrm{d}^{\prime}$ autres ont pu produire quand ils étaient en $6^{\mathrm{e}}$ (ou en seconde). Dans cette perspective, on peut aussi imaginer que l'enseignant organise une aide à la réécriture entre des élèves plus avancés dans le cursus et des plus jeunes. Le profit sera double, valorisant pour l'aîné, modélisant pour le cadet.

Ce type de réécriture permet d'instaurer des sociabilités de scripteurs et de travailler aussi bien sur des textes fictionnels que documentaires ou didactiques.

\section{La réécriture partagée}

Si la réécriture est un surtravail, alors l'allégement de la tâche peut prendre la voie d'un partage du travail scriptural. Ce partage, dont la valeur est à la fois pratique et symbolique, peut être d'un double type.

Le partage entre pairs : réécriture à plusieurs d'un seul texte que l'on décide de " reprendre », réécriture par chacun d'un même fragment de texte pour éprouver par comparaison la possibilité des différentes solutions scripturales, circulation de tous les premiers états de textes produits dans la classe et réécriture par les pairs.

Le partage entre pairs et " experts " : soumettre aux élèves des états embryonnaires ou encore peu élaborés d'un fragment d'avant-texte d'auteur et leur demander d'écrire à partir de ces données textuelles primitives une version plus achevée, proposer aux élèves d'intervenir dans le premier jet rédactionnel du texte écrit par le professeur lui-même (pour autant qu'une didactique de la réécriture implique que l'enseignant ne se mette pas hors jeu).

\section{La réécriture multipliée}

Loin d'être une étape seconde du travail, une suite et une poursuite (par d'autres moyens) d'un premier état d'un texte à améliorer en fonction d'insuffisances constatées ou d'améliorations suggérées, la réécriture peut être l'objet original et l'enjeu même du travail. En effet, comment mieux faire saisir l'espace des possibles scripturaux, des choix, des régulations, des stratégies, des marges de man$œ u v r e$, des essais, en un mot des alternatives d'écriture qu'en sollicitant des élèves la rédaction de cinq ou six versions. Ce jeu des variantes textuelles peut être le résultat de consignes qui portent sur les enjeux de communication (diversifier les destinataires d'une version à l'autre) ou sur les enjeux pragmatiques (chaque nouvelle version vise un effet discursif particulier : convaincre, amuser, expliquer, 
intriguer, etc.). Des exercices de réécriture comme autant d'Exercices de style, en somme.

Les textes de fiction tout comme les textes documentaire se prêtent à ce travail personnel ou collectif de variations scripturales.

\section{La réécriture différenciée}

Dans ce cas de figure, c'est la fonction communicationnelle du texte qui change. Certains discours manifestent une visée à dominante ethico-pratique (c'est l'écriture d'atelier), d'autres affichent une ambition esthético-formelle (c'est l'atelier d'écriture) (14). La réécriture consistera ici à passer d'un modèle discursif à l'autre. Voilà un exemple.

« Moosbrugger tira posément son coutelas de sa gaine et en éprouva le fil sur son pouce. Malgré l'âpre gel, on ne pouvait laisser le sanglier dans sa peau : il était bien trop échauffé. Le visage du chasseur prit un air ancestral, illuminé d'une sorte de ricanement solennel, qui étirait de haut en bas les rides profondes de sa face. II s'agenouilla sur l'une des pattes de derrière et empoigna l'autre de la main gauche. Puis il trancha de sa lame la peau tendue, et la fendit jusqu'au sternum. II commença par l'amputer de deux objets semblables à des œufs d'oie, d'un bleu scintillant, et les jeta derrière lui, tandis que les rabatteurs ricanaient d'un air servile :

"Attrape, renard, voilà ton dîner !"

Puis il suivit soigneusement un cordon de muscles. L'âcre fumet qui baignait la bête devint piquant ; les chasseurs reculèrent, poussant des jurons. Moosbrugger enfonça ses deux mains dans le ventre béant et remonta entre les côtes, en tira des viscères bleus et rouges, en réservant les parties nobles. " (15)

«Conseils aux veneurs pour le dépeçage

- utiliser un coutelas bien effilé ;

- ne pas laisser le sanglier abattu dans sa peau ;

- poser le genou sur l'une des pattes arrières de l'animal et saisir l'autre de la main gauche ;

- trancher la peau jusqu'au sternum ;

- castrer la bête ;

- etc. »

\section{La désécriture délibérée}

Dans sa Correspondance, Flaubert fait régulièrement des retours méta-scripturaux sur l'avancée de sa prose : "La Bovary remarche. J'attends une seconde lecture pour être convaincu que je suis dans le bon chemin. Je ne dois pas en être loin cependant [...]. Je n'ai plus guère que des difficultés d'exécution. Puis il faudra récrire le tout car c'est un peu gâché comme style. Plusieurs passages auront besoin d'être reécrits, et d'autres désécrits » (16).

En situation scolaire, la désécriture suppose que l'on retravaille des textes d'auteurs dont les caractéristiques stylistiques sont extrêmement marquées. Ainsi, un texte au lyrisme exacerbé (une page romantique par exemple) se verra délibérément dépouillé de ces marques de subjectivité pour obtenir un écrit plus neutre ( $O$ O Temps suspend ton vol... » / « II faudrait que le temps s'arrête et que

(14) Sur cette distinction dans les enjeux sociaux des textes, se reporter aux analyses de B. Lahire sur les modes pluriels d'appropriation, op.cit., p. 115.

(15) E. Jünger, Visite à Godenholm suivi de La Chasse au sanglier, Paris, 10/18, domaine étranger, 1968, pp. 122-123.

(16) Flaubert, Correspondance, II, 1851-1858, éd. de J. Bruneau, Bibliothèque de La Pléiade, nrf, Gallimard, 1980 Lettre à Louise Colet, 25 octobre 1853, pp. 457-458. 
I'on puisse profiter des moments agréables... ») ; une prose naturaliste sera désécrite pour aboutir à un récit qui gomme la violente expressivité des corps :

«Ce louchon d'Augustine, qui rôdait sournoisement autour des enfants, profitait de ça pour prendre les lardons à pleine main, sous prétexte de refaire le partage. Nana, furieuse, la mordit au poignet » / " Augustine tournait autour des enfants et prenait quantité de lardons, sous prétexte de refaire le partage. Nana était vraiment furieuse (17). »

Resterait dans cette perspective discursive à mentionner une dernière situation et un dernier enjeu de réécriture, la réécriture en abyme à l'occasion de laquelle le scripteur adopte une posture critique sinon autocritique à l'égard de sa propre prose en la parodiant ou en la pastichant : "La didactique de la réécriture suppose et impose une réécriture de la didactique (sur la méta-didactique de la réécriture et les différences historiques et théoriques avec le rewriting au sens psycho-cognitiviste du terme, voir notre article dans Pratiques) etc. ! "

Un retravail sur la production écrite suppose donc que l'institution scolaire du scripteur l'aide à se départir des mythes de l'immaculée conception rédactionnelle, ne serait-ce qu'en lui proposant régulièrement quelques-unes des situations-problèmes suggérées ici. On peut faire le pari que le transfert des représentations et des postures (se débrouiller avec les brouillons) sera favorable à un apprentissage plus systématique et plus contrôlé des techniques (sinon de l'art) de la réécriture.

D'autant que les textes électroniques vont activer encore plus systématiquement les pratiques de réécritures en décuplant les possibilités d'intervention du lecteur dans le texte. L'appropriation des textes numérisés tend en effet à effacer l'opposition entre lecture et écriture puisque sur l'écran le texte n'est pas stabilisé et peut donc être constamment modifié, au moindre coût.

Le " lecteur " post-moderne est invité à " soumettre les textes électroniques à de multiples opérations : indexer, annoter, copier, déplacer, recomposer, etc. mais plus encore il peut en devenir le coauteur. Le lecteur devant écran devient un des acteurs de l'écriture à plusieurs mains, ou tout le moins il se trouve en position de constituer un texte nouveau à partir de fragments librement découpés et assemblés [...]. Le lecteur de l'âge électronique peut construire à sa guise des ensembles textuels originaux, dont l'existence, l'organisation et l'apparence ne dépendent que de lui. Il peut à tout moment intervenir sur les textes, les modifier, il pourra les réécrire $[\ldots] »(18)$.

\section{Réécriture et fonctionnement du champ littéraire}

Mais le développement d'un comportement de scripteur devrait passer aussi par la prise de conscience des conditions institutionnelles de la réécriture. En effet, la réécriture ne procède pas toujours, tant s'en faut, d'enjeux purement esthétiques liés à l'économie interne et formelle du projet d'écriture, ni même de pratiques scripturales strictement personnelles.

Une micro-sociologie de la production littéraire se doit en effet de rappeler que nombre de textes sont retravaillés en fonction de contraintes externes, sont repris et retouchés en fonction des logiques souvent conflictuelles des agents du champ littéraire, logiques elles-mêmes différenciées selon que le produit scriptural est destiné au marché de la production restreinte, moyenne ou élargie et selon que

(17) E. Zola, L'Assommoir.

(18) G. Cavallo et R. Chartier, Histoire de la lecture dans le monde occidental, Paris, Le Seuil, 1997, p. 37 
l'écrivain est en position d'autonomie ou d'hétéronomie, en situation d'émergence, de reconnaissance ou de consécration, d'illégitimité ou de légitimité, etc.

Il y aurait beaucoup à dire et à penser, par exemple sur les réécritures des titres en fonction d'enjeux éditoriaux (manuscrit d'auteur accepté par tel éditeur dans telle collection à condition de changer le titre), sur les réécritures par autocensure du dernier état d'un avant-texte en fonction de la position que l'auteur vise à occuper dans le champ (Flaubert lui-même...), réécritures partielles ou non par concessions anticipées et parfois précipitées à la critique (nombre d'écrivains testent auprès de pairs ou d'amis, dans des salons ou dans leur salon, des états avancés de leur texte), réécritures " assistées " par des experts de la maison d'édition où le manuscrit est "retravaillé ", réécritures d'un nouveau texte en chantier pour lutter contre l'acharnement répressif de la censure et y répondre par une subversion scripturale accrue (P. Guyotat), réécritures obéissant aux cahiers des charges techniques touchant à la longueur ou à la mise en forme du texte (19), réécritures par changement de statut pragmatique d'un premier état semi-public du texte (publication élargie d'un document de littérature grise ou édition d'un texte théâtral destiné seulement aux comédiens dans un premier temps), etc. On pourrait jouer précisément avec ses élèves à imaginer, répertorier et illustrer ces situations exogènes où la réécriture s'impose à l'écrivain, volens nolens (20).

II est clair que ces réécritures ne visent pas à strictement parler à une amélioration scripturale mais plutôt à un ajustement éditorial qui est souvent en fait de l'ordre de l'adaptation (voire de la soumission, parfois aussi de la résistance) aux règles du marché littéraire.

On est loin des conceptions purement textuelles (irréalistes et idéalistes, réductrices) qui autonomisent les processus rédactionnels du champ des pratiques, de leurs règles, de leurs enjeux et donc de leurs luttes. En ouvrant le processus d'écriture sur les contraintes externes qui le travaillent, on se rapproche de ce qui construit une culture de scripteur et on rend plus complètement raison des types contextuels d'engagement dans la réécriture.

Aussi devrait-on à la fois entrer dans le détail le plus fin du retravail des réalités textuelles sans perdre de vue les réalités institutionnelles d'ensemble, articuler plus explicitement et plus systématiquement approche textuelle (l'exercice relève de la compétence du scripteur) et approche culturelle (le droit de regard est exercé par les autres agents du champ), pouvoir de réécriture et pouvoir sur la réécriture.

(19) La lecture des correspondances d'écrivains, des biographies d'éditeurs ou des témoignages de lecteurs professionnels attachés à des maisons d'édition est particulièrement riche d'informations sur ce sujet. Pour une « ap proche bibliographique » du " publiable», se reporter à J.-Y. Mollier et P. Sorel, "L'histoire de l'édition, du livre et de la lecture en France aux $\mathrm{XIX}^{\mathrm{e}}$ et $\mathrm{XX} \mathrm{X}^{\mathrm{e}}$ siècles ", Actes de la recherche en sciences sociales, dir. P. Bourdieu, Edition, Editeurs (I), 126-127, mars 1999, Seuil, pp. 39-59.

II y a tout lieu de penser que dans la mesure où de nouvelles significations dépendent directement de leurs nouvelles formes, la mise en forme éditoriale (disposition nouvelle du texte sur la page, styles de typographies adoptés, formats variables du livre, types de paratextes, etc.) produit, sur l'auteur sinon sur le lecteur, des effets connotés de réécriture. Sur le thème "les formes ont un effet sur le sens », voir D.F. McKenzie, La bibliographie et la sociologie des textes, Paris, Ed. du Cercle de la Librairie, 1991 (préface de R. Chartier).

(20) Il serait intéressant de se demander si les pratiques privées d'écriture (correspondance personnelle, journal in time, écritures ordinaires, etc.) sont plus propices ou non à la réécriture que les écrits destinés à publication. 